\title{
Predictive control of a micro bead's trajectory in a dielectrophoresis-based device
}

\author{
Mohamed Kharboutly, Michaël Gauthier and Nicolas Chaillet
}

\begin{abstract}
Micro and nano-particles can be trapped by a non uniform electric field through the effect of the dielectrophoretic force. Dielectrophoresis (DEP) is used to separate, manipulate and sense micro particles in several domains, such as in biological or Carbon Nano-Tubes (CNTs) manipulations. This paper tackles the creation of a closed loop strategy in order to control, using DEP, the trajectory of micro objects using vision feedback. A modeling of the dielectrophoresis force is presented to illustrate the non linearity of the system and the high dynamics of the object under dielectrophoresis . A control strategy based on the generalized predictive control method is proposed with the aim of controlling the trajectory, taking advantage of the high dynamics despite the non linearity. Simulated results are shown to evaluate our control strategy.
\end{abstract}

\section{INTRODUCTION}

In order to increase the functionalities and the density of microelectronic devices and microsystems, 3D integration is becoming a great challenge. The general principle is to build a system based on the vertical assembly of planar dies. Industrial trend consists in using smaller and smaller dies in order to reduce the size of the final systems [1]. Currently, die to die assembly requires new packaging methods able to assemble micro-parts whose sizes are typically around $200 \mu \mathrm{m}$ with a typical accuracy of $1 \mu \mathrm{m}$ and a high throughput. Two ways are usually proposed in micro-assembly: (i) the robotic assembly which consists in handle and assemble the object using microtweezers [2], [3], [4] and (ii) the self-assembly where the object trajectory is driven by long range forces (capillary force or dielectrophoresis force) [5], [6] without adhesion disturbance and with a high throughput. The present paper is focused on the improvement of dielectrophoresis self-assembly. Current dielectrophoresis devices are only controlled in open loop, such as the manipulation of particles [7], continuous separation [8] and analysis of micro particles [9] like biological cells and bacteria, dielectrophoretic fieldflow fractionation (DEP-FFF) separation [10], positioning and sensing [11] micro particles and translation motion of carbon nanotubes [12]. As these micro manipulation devices are controlled in open loop, the object trajectory and the

M. Kharboutly, Member, IEEE is with FEMTO-ST Institute CNRS UFC/ENSMM/UTBM Automatic Control and Micromechatronic Systems Department, 24 rue Alain Savary, 25000 Besançon, France mohamed.kharboutly@femto-st. fr

M. Gauthier, Member, IEEE is with FEMTO-ST Institute CNRS UFC/ENSMM/UTBM Automatic Control and Micromechatronic Systems Department, 24 rue Alain Savary, 25000 Besançon, France michael.gauthierefemto-st. fr

N. Chaillet, Member, IEEE is with FEMTO-ST Institute CNRS UFC/ENSMM/UTBM Automatic Control and Micromechatronic Systems Department, 24 rue Alain Savary, 25000 Besançon, France nicolas.chailletefemto-st.fr final position are thus not guaranteed. This paper deals with a strategy able to control the high-speed object trajectory in a dielectrophoresis device using vision feedback. In order to simplify the presentation of the control approach, this paper is focusing on the positioning of a micro bead.

The behavior of a micro bead under dielectrophoresis is characterized by its non linearity and its high dynamics compared to the vision speed rate. In this paper, we propose and study a control strategy based on a generalized predictive control enabling the reference's tracking.

In the next section, we introduce the dielectrophoresis force and present the dynamic model of the micro bead in the dielectrophoresis-based device. Secondly the behavior of the micro bead is studied and the corresponding issues are presented.

In the third section, a linearization of the model is proposed, generalized predictive control strategy is introduced and its application on our model is demonstrated.

The final section presents several simulated results and discussions.

\section{MODEL PRESENTATION}

\section{A. Dynamic Model}

The general expression of the dielectrophoretic force, created by a non uniform electric field, applied to a micro bead submerged in a liquid medium is [13], [14] is:

$$
\overrightarrow{F_{D E P}}=2 \pi \epsilon_{0} \epsilon_{p} r^{3} \operatorname{Re}[K(\omega)] \overrightarrow{\nabla \mathbb{E}^{2}} .
$$

$K(\omega)$ is the Clausius - Mossotti factor:

$$
K(\omega)=\frac{\epsilon_{p}^{*}-\epsilon_{m}^{*}}{\epsilon_{p}^{*}+2 \epsilon_{m}^{*}},
$$

and

$$
\epsilon^{*}=\epsilon+\frac{\sigma}{j \omega},
$$

where $\epsilon$ are the permittivities, $\sigma$ are the conductivities, index $O$ refers to the vacuum, index $m$ refers to the medium and index $p$ refers to the micro bead, $\mathrm{r}$ is the radius of the micro bead, $\omega$ is the angular frequency of the applied electric field, $\vec{\nabla}$ is the gradient operator and $\mathbb{E}$ is the root mean square magnitude of the sinusoidal electric field.

The electric field $\mathbb{E}$ is created by applying an electric voltages on pattern of electrodes as described in Fig. 1.

The dynamic model of the micro bead is defined by the Newton second's law. The force applied to the micro bead are the dielectrophoresis force, the Stokes drag force $\overrightarrow{F_{d r a g}}$ and its own weight $\vec{P}$ (see Fig.1). 


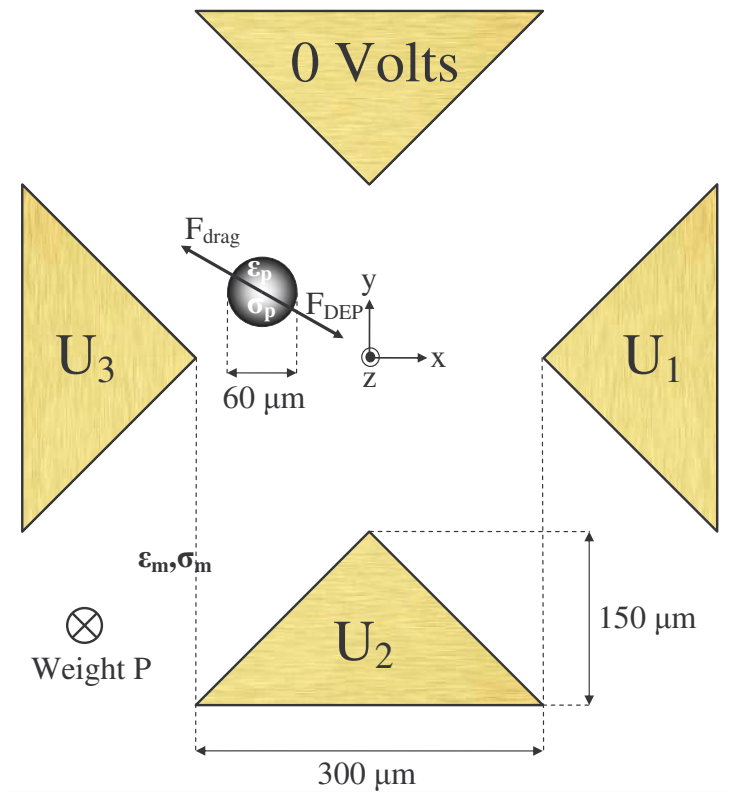

Fig. 1. DEP-based device used in this study

If we consider that the position $X(x, y, z)$ of the micro bead is defined by its center's coordinates, thus the $\vec{X}$ is the velocity of the particle and the $\overrightarrow{F_{d r a g}}$ verifies:

$$
\overrightarrow{F_{\text {drag }}}=-6 \pi \mu r \vec{V}=-k_{\mu} \overrightarrow{\dot{X}}
$$

where $\mu$ is the dynamic viscosity of the liquid medium. Using Newton's second law the particle's motion is defined by:

$$
\overrightarrow{F_{D E P}}+\vec{P}-k_{\mu} \overrightarrow{\dot{X}}=\mathcal{M} \overrightarrow{\ddot{X}}
$$

where $\mathcal{M}$ is the mass of the micro bead and $\overrightarrow{\ddot{X}}$ is the acceleration vector. We have shown in [15] that, in this situation, the inertial term $\mathcal{M} \vec{X}$ is a negligible volumic effect in the micro-world : the respond time corresponding to the acceleration term is negligible compared to the respond time corresponding to the fluid dynamic term. Thus, the particle's motion equation can be reduced as follows:

$$
\overrightarrow{\dot{X}}=\frac{\left(\overrightarrow{F_{D E P}}+\vec{P}\right)}{k_{\mu}}
$$

A voltage vector $U=\left[U_{1}, U_{2}, U_{3}\right]$ applied on the electrodes creates the non uniform electric field $\vec{E}$ which creates the dielectrophoresis force used to manipulate the micro particle. Equation (6) manages the dynamical behavior of the micro particle under dielectrophoresis force.

\section{B. Study of the micro bead behavior}

In order to present our control strategy, we are focusing on the electrode's geometry described in Fig. 1 submerged in ultra pure water. We assume here that the micro bead only moves along the $x$ axis, thus the position $X$ of the micro bead is defined by $(x, y=0, z=r)$. Projecting (6) along the $x$ axis, the velocity of the micro bead is ruled by:

$$
\dot{x}=\frac{F_{D E P}(x, U)}{k_{\mu}}
$$

In order to maintain the micro bead's center along the $x$ axis, and taking into consideration the electrodes symmetry, the control input vector, which is the applied voltage vector $U$, proposed here is:

$$
U=\left[U_{\text {ref }}-\delta u, 0 V, U_{r e f}+\delta u\right] .
$$

where $U_{\text {ref }}$ is a fixed voltage, in this study it is equal to $75 \mathrm{~V}$, and $\delta u$ is the single control variable. The electric field and the applied voltage on the electrodes are linearly related, due to the electrostatic superposition principle and the proportional relation between the electric potential and the charge density:

$$
\mathbb{E}=a(x)\left(U_{\text {ref }}-\delta u\right)+b(x)\left(U_{\text {ref }}+\delta u\right) .
$$

This relation allows to replace the electric field $\mathbb{E}$ in (1) by a linear combination of the applied voltages. Thus, from (7) the velocity $\dot{x}$ can be written as a second degree equation, coming from the electric field's square in the dielectrophoresis equation (1), with the respect to the control variable $\delta u$ :

$$
\dot{x}=f_{1}(\delta u)=\alpha(x) \delta u^{2}+\beta(x) \delta u+\gamma(x)
$$

where $\alpha(x), \beta(x)$ and $\gamma(x)$ characterize the dynamic model. They are function of the state variable $x$. The first problem to control this system is its non linearity which is shown in the equation (10).

The first non linearity of the system with respect to the control variable $\delta u$ is due to the square term $\delta u^{2}$.

The second non linearity comes from the non linearity of the functions $\alpha(x), \beta(x)$ and $\gamma(x)$. These functions characterize the system and they are identified using the hybrid simulation method, described in [15], which combines preprocessing FEM software simulated data and analytic equations.

Fig. 2 shows the non linearity of these functions. In this figure, we clearly see that the functions $\alpha(x), \beta(x)$ and $\gamma(x)$ are not linear with respect to the state variable $x$. This non linearity increases as the distance between the micro bead and the electrode's edge decreases.

Moreover, the micro bead reaches high speed motion when applying high voltages. Fig.3 shows the step response for a micro bead starting from the initial position $x_{0}=0 \mu \mathrm{m}$ and applying a voltage of $\delta u=70 \mathrm{~V}, 60 \mathrm{~V}, 50 \mathrm{~V}$ and $40 \mathrm{~V}$. If we compare the time constant of the micro bead's response, which is close to $3 \mathrm{~ms}$, to a relatively high speed camera of 400 ips (images per second) we can note that during this time only two positions can be measured.

\section{Control StRATEgy}

In order to control the micro bead's trajectory along a reference trajectory $w$ in a dielectrophoresis-based device using vision feedback, two main difficulties occur.

The first problem is the non linearity of the system with respect to the control variable $\delta u$ as the equation (10) shows and the non linearity in relation to the state variable $x$ due 


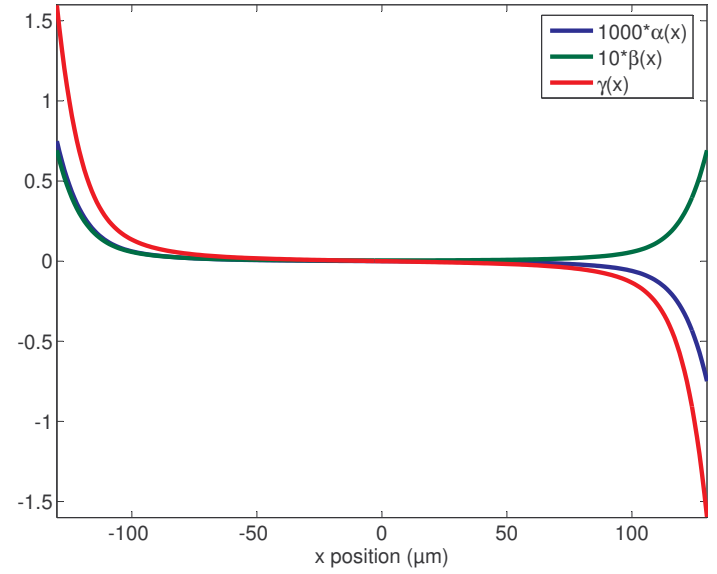

Fig. 2. The non linearity of the three functions $\alpha(x), \beta(x)$ and $\gamma(x)$ $\left(U_{\text {ref }}=75 \mathrm{~V}\right)$ especially when $x \geq 50 \mu \mathrm{m}$, respectively expressed in $m s^{-1} V^{-2}, m s^{-1} V^{-1}$ and $m s^{-1}$.

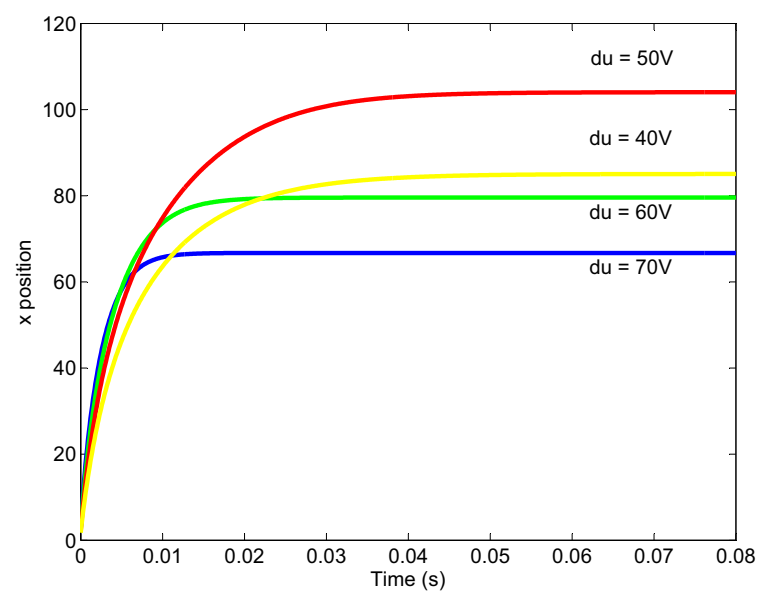

Fig. 3. Step response for a micro bead starting form $x_{0}=0 \mu m, \delta u=$ $40 \mathrm{~V}, 50 \mathrm{~V}, 60 \mathrm{~V}$ and $70 \mathrm{~V}$ and $U_{\text {ref }}=75 \mathrm{~V}$

to the non linearity of the functions $\alpha(x), \beta(x)$ and $\gamma(x)$ as it is shown in Fig.2.

Moreover, the other problem is the high dynamics of the system which induces high speed motion of the micro bead compared to the camera speed rate, which is one of the most conventional way to measure the micro bead's position.

Both non linearity and high dynamics led us to develop an appropriate control strategy (Fig.4).

\section{A. Linear model}

To resolve the non linearity problem, starting by the non linearity relative to the control variable $\delta u$, the first step consists of transforming this non linear system to another linear system relatively to a new control variable named $\xi$. Using the following variable transformation:

$$
\xi=f_{2}(\delta u)=\left(\delta u+\frac{\beta(x)}{2 \alpha(x)}\right)^{2},
$$

we are able to create a linear relation between the new control variable $\xi$ and the velocity of the micro bead $\dot{x}$, respecting

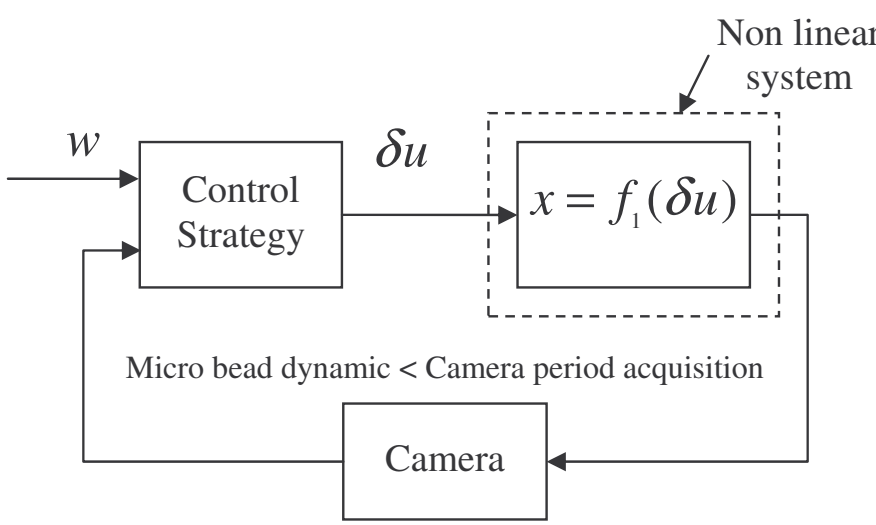

Fig. 4. Summary of the control strategy

the constraint $C_{1}$ :

$$
\xi \geq 0
$$

The new linear dynamic equation is:

$$
\dot{x}=\alpha(x) \xi+\rho(x),
$$

where

$$
\rho(x)=\gamma(x)-\frac{\beta^{2}(x)}{4 \alpha(x)} .
$$

In the case of $\alpha(x)$ is equal to 0 :

$$
\xi=\beta(x) \delta u+\gamma(x) .
$$

Equation (13) solves the non linearity problem in relation to the new control variable $\xi$.

Concerning the non linearity in relation to the state variable, produced by the non linear functions $\alpha(x), \beta(x)$ and $\gamma(x)$ (see Fig.2), these functions can be estimated by using an estimated value of the state variable $x$. This estimated value is equal to the current position when it is available, and it is equal to the reference value $w$ date when the state variable $x$ is not available. This last case is based on the hypothesis that the reference trajectory is known at any time and the controlled position is relatively close to the desired position.

\section{B. Generalized Predictive Control (GPC)}

In order to control the high dynamics of the micro bead, a control strategy ables to apply a series of control variables while no position's informations are available between two successive camera acquisition is presented. One of the control strategy which fulfill these requests is the GPC.

The goal of the generalized predictive control is to find the optimal future control actions that drive the future process output to track the reference trajectory as closely as possible in the presence of system constraints and disturbances [16]. The generalized predictive control is used in several domains of applications such as solar power plants [17], turbine engines [18] and robotic manipulators [19]. The main idea of the GPC is to find a future control sequence from a given 
time which minimizes the error between the predicted output and the reference.

Based on a numerical model, the GPC enables to calculate the optimal control sequence of $N$ values $\xi$ in the future which minimize the error between the output position and the reference $w$ in $N$ steps in the future.

The application of the GPC strategy on our system requires a discrete model. Considering that the camera acquisition period is $T_{c}$ which means the position's information is updated each $T_{c}$ seconds. During this period the controller calculates the appropriate control variable sequence of $N$ values using the sample time $T_{s}$ in order to track the reference trajectory (see Fig.5) with $N \times T_{s} \geq T_{c}$.

The minimization process consists of minimizing a criterion $J$ with respect to the the sum of the errors' square between the predicted position and the reference. This criterion is based on a numeric model of the system as the following:

$$
J=\sum_{i=1}^{N}\left(x_{i, j}-w_{i, j}\right)^{2}+\sum_{i=1}^{N} \lambda \xi_{i-1, j}^{2}
$$

with respect to the constraint $C_{1}$ (12) where:

1) $N$ is the prediction's horizon,

2) index $i, j$ represent the time $j \times T_{c}+i \times T_{s}$ (see Fig.5) and

3) $\lambda$ is a weighting affecting the control variable $\xi$.

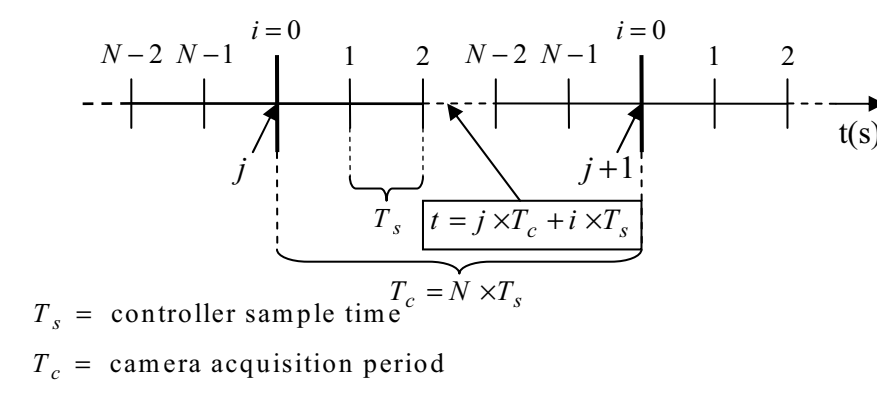

Fig. 5. The two samples time: camera acquisition period $T_{c}$ and controller sample time $T_{s}$

$x_{i, j}$ can be calculated after discretization (13) using the sample time $T_{s}$ and the camera period $T_{c}$ as the following:

$$
x_{i, j}=T_{s}\left[\alpha\left(x_{i-1, j}\right) \xi_{i-1, j}+\rho\left(x_{i-1, j}\right)\right]+x_{i-1, j} .
$$

The solution of the criterion $J$ is the optimal control variable sequence which minimizes the error between the output position and the reference in $N$ steps in the future with respect to position at the date $j$ considered as the initial position $x_{0, j}$.

Then, the criterion $J$ is solved, at each time of the camera acquisition $\left(j \times T_{c}\right)$, iteratively by nullifying its derivate with respect to the control variables $\xi_{i, j}$, where $1 \leq i<N-1$.

The nullification of the criterion's derivate with respect to the control variable $\xi_{i, j}$ gives:

$$
\frac{\delta J}{\delta \xi_{i, j}}=g_{i}\left(\xi_{0, j} \ldots \xi_{N-1, j}\right) \times \xi_{i, j}=0 .
$$

The first solution of (18) occurs when $g_{i}\left(\xi_{0, j} \ldots \xi_{N-1, j}\right)=$ 0 . This solution minimizes the criterion to 0 . Thus, we can prove that the $l t h$ control variable value $\xi_{i, j}$ of the $j t h$ optimal sequence which minimize the criterion at the date $i, j$ is equal to:

$$
\begin{aligned}
\xi_{i, j}= & \frac{1}{\alpha\left(x_{i, j}\right)}\left[\frac{-\lambda}{2 \alpha\left(x_{i, j}\right)}+\frac{\lambda}{2 \alpha\left(x_{l+1, j}\right)}\right]+ \\
& \frac{1}{\alpha\left(x_{i, j}\right)}\left[-\rho\left(x_{i, j}\right)+w_{i, j}-x_{i, j}\right]
\end{aligned}
$$

To calculate the first control variable value $\xi_{0, j}$, the position $x_{0, j}$ and $x_{1, j}$ are required. The position $x_{0, j}$ is the measured position by the camera at the date $j$ but the position $x_{1, j}$ is not calculated yet, thus it is estimated by the reference position $w_{1, j}$.

Then the next state variable $x_{1, j}$ is calculated using (17). We proceed by doing the same iteration until we calculate the $N$ control variables which minimize the criterion $J$ at the date $j$.

If the calculated value of $\xi_{i, j}$ does not respect the constraint $C_{1}$ then $\xi_{i, j}$ takes the value 0 which is the other solution of (18) and it minimizes the criterion $J$ to a value different to 0 .

By minimizing the criterion $J$ at the time $j \times T_{c}$ we obtain a sequence of $N$ values of the control variable $\xi_{i, j}$. Using this sequence, we calculate the corresponding values of the initial control variable $\delta u_{i, j}$ using $\delta u_{i, j}=f_{2}^{-1}\left(\xi_{i, j}\right), f_{2}$ being given in (11), which will be applied to the system.

The diagram given in Fig.6 illustrates the control scheme where the GPC, based on the linear model, is used to control the position of the micro bead by calculation of the optimal sequence of the control variable $\xi$. After that, the real control variables $\delta u$ are calculated using the function $f_{2}^{-1}$ from (11). Then we apply this sequence of the control variable $\delta u$ to the non linear system. When a new measurement is available from the camera then a new sequence of the control variable $\xi$ is calculated with respect to the new measured position $x_{0, j}$ considered as the initial position in the next iteration.

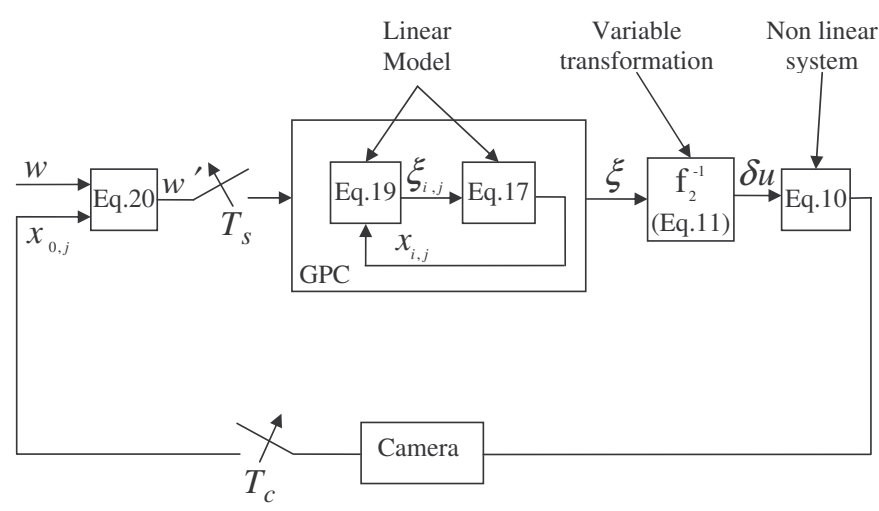

Fig. 6. Bloc diagram presents the principal difficulties to control the system

In order to avoid large variations of the applied voltage especially after a new camera acquisition when the error between the real position and the reference could be large, 
we add a correction term $e_{i, j}$ to the reference position $w_{i, j}$ to obtain a modified reference trajectory $w_{i, j}^{\prime}$. The variable $e_{i, j}$ is calculated with respect to the date $i$ and the measured position $x_{0, j}$, so that $e_{i, j}$ decreases when $i$ increases as shown in Fig.7. The term $e_{i, j}$ can be presented as the following:

$$
e_{i, j}=\frac{N-i}{N}\left(w_{i, j}-x_{0, j}\right)
$$

and

$$
w_{i, j}^{\prime}=w_{i, j}-e_{i, j}
$$

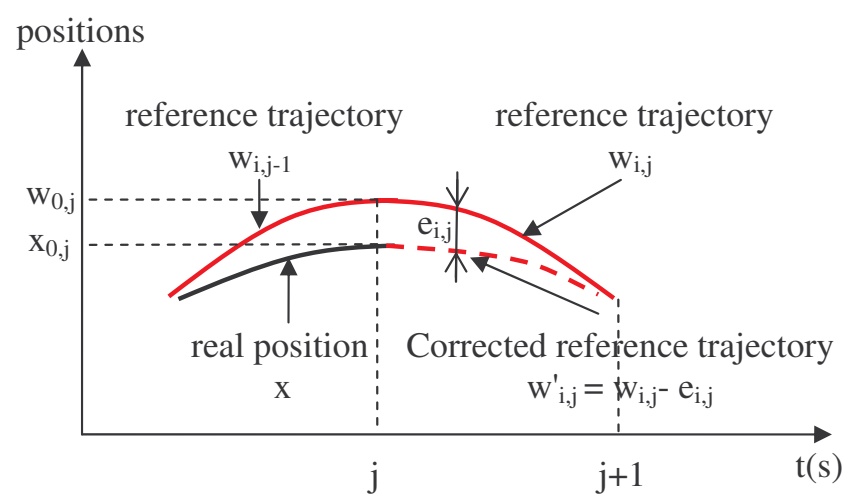

Fig. 7. New trajectory $w_{i, j}^{\prime}$ in function of the position $x_{0, j}$ and $w_{i, j}$

\section{RESULTS AND DISCUSSION}

In order to test the proposed control strategy, the dielectrophoresis system described in Fig.1 has been simulated, where the liquid medium is ultra pure water with $\epsilon_{m}=80 \epsilon_{0}$, $\sigma_{m}=10^{-16} \mathrm{Sm}^{-1}$ and $\mu=10^{-3} \mathrm{~kg}(\mathrm{sm})^{-1}$. The micro object is a silicium micro bead with radius $r=30 \mu \mathrm{m}$, $\epsilon_{p}=8.4 \epsilon_{0}$ and $\sigma_{p}=10^{-12} \mathrm{Sm}^{-1}$. The frequency $2 \pi \omega$ of the applied voltage used to create the non uniform electric field is $10 \mathrm{kHZ}$ and $U_{\text {ref }}=75 \mathrm{~V}$ and the applied voltage on the electrodes is limited to $U_{\max }=150 \mathrm{~V}$.

The sample time is chosen equal to $0.5 \mathrm{~ms}$ and the camera has an acquisition sample time equal to $2.5 \mathrm{~ms}$. Thus, the minimum value of the prediction's horizon $N$ is equal to $2.5 / 0.5=5$ steps.

In order to test the robustness of the control law, the model used in the GPC controller and the simulated model differs by adding errors of $20 \%$ on the electric permittivities of both medium and particle.

\section{A. High dynamics}

Firstly, the proposed control strategy has been tested on high dynamic reference trajectories. Considering a sinusoidal reference trajectory with period equal to 10 times the camera acquisition period, i.e. $25 \mathrm{~ms}$ with a magnitude of $25 \mu \mathrm{m}$ around $x=0$. In this range the model can be considered linear (see Fig.2).

Fig. 8 shows the output position of the the micro bead's calculated by the real system using the control variable $\delta u$ obtained from the control variable $\xi$ calculated by the proposed GPC applied on the model. This control strategy is also compared to a regular PI corrector to demonstrate the efficiency of our strategy. The proportional constant of this PI corrector is equal to the inverse of the gain of the system considered as a first order linear system in this range. The gain of the system is calculated and it is equal to $1.610^{-6} \mathrm{mV}^{-1}$. The integrator constant of the PI corrector is equal to the time constant of the system which is equal to $3.610^{-3} s$.

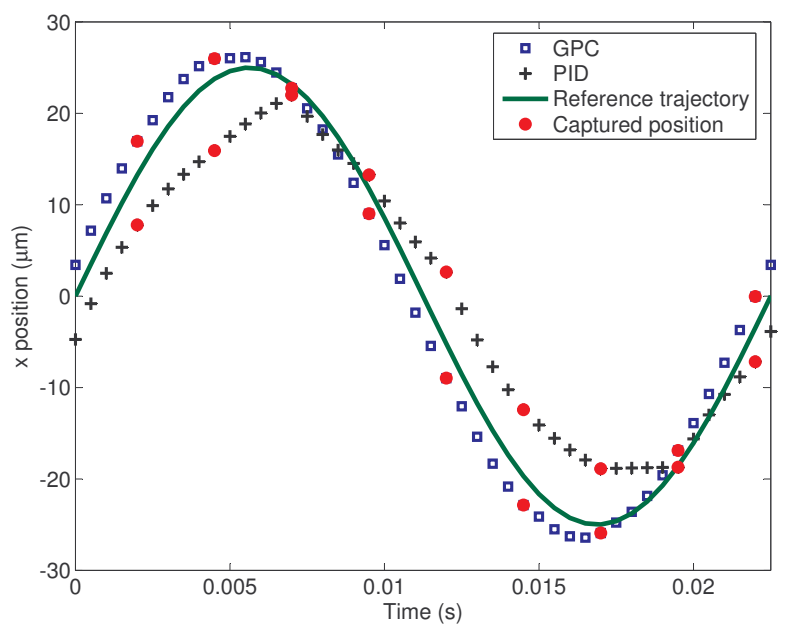

Fig. 8. Output trajectory of the system controlled by the generalized predictive control and compared to the PI control. The camera acquisition period is $2.5 \mathrm{~ms}$

\section{B. Non linearity}

Secondly, we test the proposed control strategy in the non linear range, by tracking a trajectory which reaches position near the electrode's edges. In this case the sinusoidal reference trajectory changes in magnitude and period.

Fig.9 shows the output trajectory of the real system controlled by the proposed GPC strategy based on the model where the amplitude of the reference trajectory is $130 \mu \mathrm{m}$ and its period is $100 \mathrm{~ms}$.

In this example, the micro bead goes toward the electrodes. At the time $t=0.01 \mathrm{~s}$, the micro bead's position is near to $x=100 \mu m$, the control did not find any value of the control variable $\xi$ with respect to the constraint $C_{1}$ and nullify the error between the calculated and the reference position. Thus, the control variable $\xi$ takes the value of 0 as explained above. In this case the applied voltage on the electrode is not necessarily at its saturation value $\left(U_{\text {ref }}+\delta u=150 \mathrm{~V}\right)$ but it takes a different value which minimizes the criterion $J(16)$ and also maximize the micro bead's velocity. This is due to the non linearity of the model near to the electrodes, where the dielectrophoresis force is not maximal for the maximal applied voltage. In Fig.10, the calculated value of $\delta u$ during the trajectory tracking is shown. At the same time, $t=0.01 \mathrm{~s}$, the controller determines $\delta u=50 \mathrm{~V}$ as the optimum value that minimizes the criterion $J$ which is 
less than the maximum allowed value $U_{\max }-U_{\text {ref }}=75 \mathrm{~V}$.

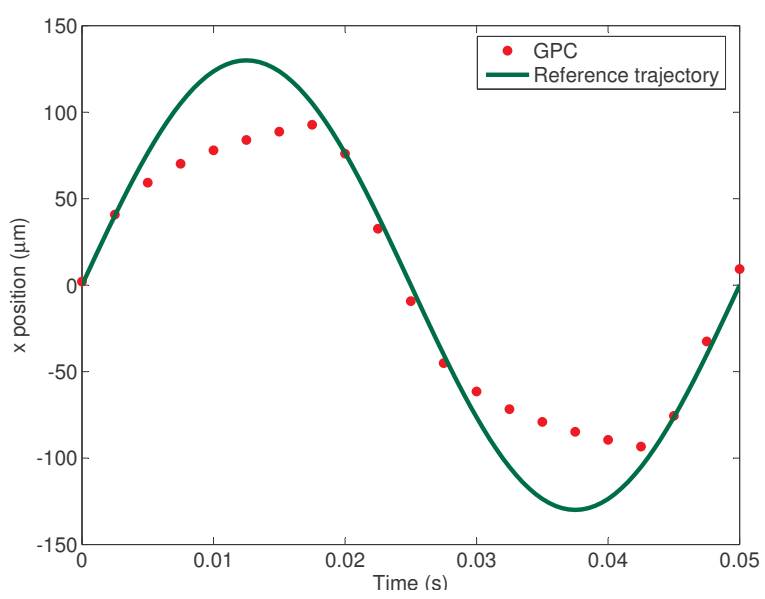

Fig. 9. Output trajectory of the Generalized predictive control tracking a long range reference trajectory using camera with acquisition period equal to $2.5 \mathrm{~ms}$.

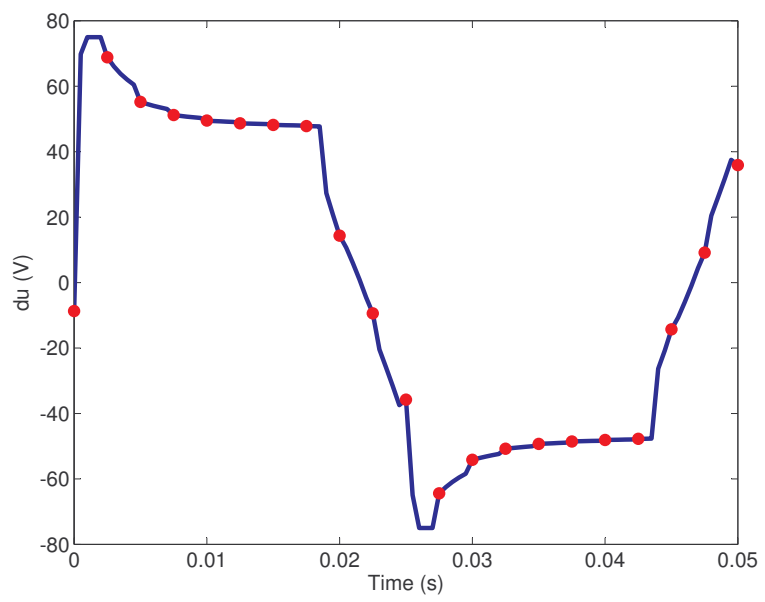

Fig. 10. Calculated value of the control variable $\delta u$ during the trajectory monitoring. The saturation value is less then the maximum allowed value $(75 \mathrm{~V})$.

\section{Conclusions}

We have proposed a closed loop control strategy based on the generalized predictive control for a dielectrophoreticbased device. The behavior of a micro bead, driven by dielectrophoresis force, is characterized by its high dynamics compared to the capture speed rate and the non linearity of its dynamic equation in relation to both the voltage variable and the position. The control strategy proposed provides the optimal sequence of voltage values with a smaller sampling rate then the camera speed rate. It enables to minimize the error between the micro bead's position and the reference even when the micro bead is near the electrodes where the non linearity is strong. The proposed control strategy is tested and compared to other regular control strategy such as the PI controller and several results are presented. These work opens the way to the closed-loop of several non contact micromanipulations whose behavior is usually similar.

\section{REFERENCES}

[1] ITRS roadmap. International technology roadmap for semiconductors. Online http://www.itrs.net/reports.html, 2008.

[2] J. Dejeu, M. Gauthier, P. Rougeot, and W. Boireau. Adhesion forces controlled by chemical self-assembly and $\mathrm{ph}$, application to robotic microhandling. ACS Applied Materials \& Interfaces, 1, no 9:96673, 2009.

[3] M. Savia and H. Koivo. Contact micromanipulation-survey of strategies. EEE/ASME Transactions on Mechatronics, 14, no. 4:504-514, 2009.

[4] H. Xie, S. Haliyo, and S. Rgnier. A versatile atomic force microscope for 3d nanomanipulation. Nanotechnology, 2:215301, 2009.

[5] M. Mastrangeli, W. Ruythooren, C. van Hoof, and J.-P. Celis. Characterization of interconnects resulting from capillary die-to-substrate self-assembly. In Proc. 2nd IEEE Electronics SystemIntegration Technology Conference (ESTC2008), pages 135-140, Greenwich, UK, 2008.

[6] Y. Yamada T. Konno J. Liang K. Sasaki K. Inamura T. Tanaka M. Koyanagi T. Fukushima, H. Kikuchi. New three-dimensional integration technology based on reconfigured wafer-on-wafer bonding technique. In Proc. IEEE International Electron Devices Meeting, pages 985-8, Washington D. C., USA, 2007.

[7] N. Aubry J. Kadaksham, P. Singh. Manipulation of particles using dielectrophoresis. Mechanics Research Communications, 33:108 122 , july 2006.

[8] Kwan Hyoung Kang, Yuejun Kang, Xiangchun Xuan, and Dongqing Li. Continuous separation of microparticles by size with direct currentdielectrophoresis. Electrophoresis, 27:694702, 2006.

[9] P. Gascoyne and J. Vikoukal. Dielectrophoresis-based sample handling in general-purpose programmable diagnostic instruments. IEEE Proceedings, (1):22 - 42, 2004.

[10] Tzon-Shyng Leu and Chih-Yuan Weng. Dynamics of dielectrophoretic field-flow fractionation (dep-fff) based micro sorter for cell separation. Modern Physics Letters B, 23(3):389-392, 2009.

[11] Liangbin Pan, Guangxin Xiang, Lihua Huange, Zhongyao Yu, Jing Cheng, Wanli Xing, and Yuxiang Zhou. Automatic positioning and sensing microelectrode array (apsmea) for multi-site electrophysiological recordings. Neuroscience Methods, 170:123129, 2008.

[12] Anoop Kumar Srivastava, Seok Jin Jeong, Myong-Hoon Lee, and Seung Hee Lee. Dielectrophoresis force driven dynamics of carbon nanotubes in liquid crystal medium. Journal of Applied Physics, 102:043503, 2007.

[13] F. T. Chang, Y. C. Lee, and C. C. Chiu. Multiple electrodes arrayeed dielectrophoretic chip with application on micro-bead manipulation. In Proceedings of the 3rd IEEE Int. Conf. on Nano/Micro Enhineered and Molecular Systems, pages 850 - 853, Sanya, China, january 2008.

[14] P. R. C. Gascoyne and J. V.Vykoual. Dielectrophoresis-based sample handling in general-purpose programmable diagnostic instruments. IEEE Sensors Journal, 92(1):22 - 42, January 2004

[15] Mohamed Kharboutly, Michael Gauthier, and Nicolas Chaillet. Modeling the trajectory of a micro particle in a dielectrophoresis device. Journal of Applied Physics, 106:114312, 2009.

[16] Yasuki Kansha and Min-Sen Chiu. Adaptive generalized predictive control based on jitl technique. Journal of Process Control, 19, Issue 7:1067-1072, July 2009.

[17] Manuel Gálvez-Carrillo, Robin De Keyser, and Clara Ionescu. Nonlinear predictive control with dead-time compensator: Application to a solar power plant. Solar Energy, 83, Issue 5:743-752, 2009.

[18] Junxia Mu, David Rees, and G.P. Liu. Advanced controller design for aircraft gas turbine engines. Control Engineering Practice, 13, Issue 8:1001-1015, 2005.

[19] F. Temurtas, H. Temurtas, and N. Yumusak. Application of neural generalized predictive control to robotic manipulators with a cubic trajectory and random disturbances. Robotics and Autonomous Systems, 54, Issue 1:Pages 74-83, 2006. 